\title{
Nivel de conocimiento sobre el manejo de emergencias médicas de los alumnos de quinto y sexto año de la Facultad de Odontología de la Universidad Autónoma del Paraguay Pierre Fauchard
}

\author{
Level of Knowledge about Medical Emergency Management by \\ Fifth- and Sixth-Year Students from the School of Dentistry \\ at the Universidad Autónoma del Paraguay Pierre Fauchard
}

Mario Martín Casco Florentín, Od. ${ }^{1}$, Ninfa Lucía Jacquett Toledo $\bowtie$, Od. $^{2}$

1 Universidad Autónoma del Paraguay Pierre Fauchard, Asunción, Paraguay

2 Universidad Autónoma del Paraguay Pierre Fauchard, Asunción, Paraguay

Universidad Autónoma del Paraguay Pierre Fauchard, Facultad de Odontología. Dirección: Colón 658-Gral. Díaz, Asunción, Paraguay. Correo electrónico: ninfajacquett@gmail.com

Recibido: 17 de abril del 2017 Aprobado: 15 de mayo del 2017

Cómo citar este artículo: Casco-Florentín MM, Jacquett-Toledo NL. Nivel de conocimiento sobre el manejo de emergencias médicas de los alumnos de quinto y sexto año de la Facultad de Odontología de la Universidad Autónoma del Paraguay Pierre Fauchard. Rev Nac Odontol. 2018;13(26):1-16. doi: http://dx.doi.org/10.16925/od.v13i26.2041

\section{Resumen}

Introducción: las emergencias médicas son situaciones que tienen consecuencias inmediatas y son riesgosas para la vida, por lo cual requieren acción rápida y precisa.

Objetivo: determinar el nivel de conocimiento de los alumnos de quinto y sexto año de la Facultad de Odontología de la Universidad Autónoma del Paraguay Pierre Fauchard sobre el manejo de situaciones de emergencias médicas que se pueden presentar durante la consulta.

Métodos: el estudio fue de tipo observacional descriptivo de corte transversal, se realizó una encuesta para evaluar los conocimientos sobre emergencias médicas a través de un cuestionario estructurado, con veinticinco preguntas cerradas de opción múltiple para medir las variables establecidas. La muestra estuvo conformada por 146 alumnos de quinto y sexto. Las variables de interés fueron: edad, sexo, participación en cursos (teórico o teórico-práctico) de primeros auxilios y nivel de conocimiento favorable o desfavorable, con base en la escala del $60 \%$ en la que se considera favorable un total de quince respuestas correctas.

Resultados y conclusiones: se concluye que el nivel de conocimiento de los alumnos de quinto y sexto año de la Facultad de Odontología Pierre Fauchard fue desfavorable en un 56\%, en una población mayoritariamente femenina con un porcentaje de $87,7 \%$, con una franja etaria de 23 años con un porcentaje de $35,6 \%$. Solo el $17,8 \%$ de los alumnos participó en algún curso de primeros auxilios, de los cuales el 6,8\% fue teórico y el 11,0\% fue teórico-práctico.

Palabras clave: alumnos, conocimiento, emergencia, odontología. 


\title{
Level of Knowledge about Medical Emergency Management by Fifth- and Sixth-Year Students from the School of Dentistry at the Universidad Autónoma del Paraguay Pierre Fauchard
}

\author{
Abstract \\ Introduction: Medical emergencies are events that have immediate consequences and are life threatening, \\ for which they require fast and accurate action. \\ Aim: To determine the level of knowledge of fifth- and sixth-year students from the School of Dentistry at the \\ Universidad Autónoma del Paraguay Pierre Fauchard on the management of medical emergencies that may \\ arise during consultation.
}

Materials and methods: Cross-sectional, descriptive, observational study. A survey was conducted to test their knowledge about medical emergencies through a structured questionnaire, with 25 multiple choice closed-ended questions to measure the established variables. The sample consisted of 146 fifth- and sixthyear students. The variables of interest were age, sex, participation in first aid courses (theoretical or theoretical-practical) and level of favorable or unfavorable knowledge, based on a $60 \%$ scale in which a total of 15 correct answers is considered favorable.

Results and conclusions: It is concluded that the level of knowledge of fifth- and sixth-year students from the School of Dentistry at Pierre Fauchard was unfavorable in $56 \%$, in a predominantly female population with a percentage of $87.7 \%$, and an age group of 23 years old with a percentage of $35.6 \%$. Only $17.8 \%$ of the students participated in a first aid course, of which $6.8 \%$ was theoretical and $11.0 \%$ was theoretical-practical.

Keywords: students, knowledge, emergency, dentistry.

\section{Nível de conhecimento sobre a gestão de emergências médicas dos estudantes de quinto e sexto ano da Faculdade de Odontologia da Universidad Autónoma del Paraguay Pierre Fauchard}

\section{Resumo}

Introdução: as emergências médicas são situações que têm consequências imediatas e que trazem riscos para a vida, por isso requerem ação rápida e precisa.

Objetivo: determinar o nível de conhecimento dos estudantes de quinto e sexto ano da Faculdade de Odontologia da Universidad Autónoma del Paraguay Pierre Fauchard sobre a gestão de situações de emergências médicas que podem ocorrer durante a consulta.

Métodos: este estudo foi de tipo observacional descritivo, de corte transversal; realizou-se uma enquete para avaliar os conhecimentos sobre emergências médicas por meio de um questionário estruturado, com 25 perguntas fechadas, de múltipla escolha, para mediar as variáveis estabelecidas. A amostra esteve conformada por 146 estudantes de quinto e sexto ano. As variáveis de interesse foram: idade, sexo, participação em cursos (teórico ou teórico-prático) de primeiros-socorros e nível de conhecimento favorável ou desfavorável, com base na escala de 60\%, na qual se considera favorável um total de 15 respostas corretas.

Resultados e conclusões: conclui-se que o nível de conhecimento desses estudantes foi desfavorável em $56 \%$, numa população predominantemente feminina, com uma porcentagem de $87,7 \%$, com faixa etária de 23 anos com uma porcentagem de $35,6 \%$. Somente $17,8 \%$ dos estudantes participaram de algum curso de primeiros-socorros, dos quais 6,8\% foram teóricos e $11 \%$ foram teórico-práticos.

Palavras-chave: alunos, conhecimento, emergência, odontologia. 


\section{Introducción}

Las emergencias médicas son definidas por la Organización Mundial de la Salud (oms) como situaciones que tienen consecuencias inmediatas y son riesgosas para la vida [1], requieren acción rápida y precisa [2-5], y pueden ocurrir en cualquier momento, en cualquier lugar y a cualquier persona. Las situaciones de emergencia es más probable que se produzcan dentro del consultorio dental, debido al mayor nivel de estrés al momento de la consulta. Por ejemplo, el miedo y la ansiedad pueden hacer que estos pacientes sean propensos a sufrir síncope e hiperventilación [6,7].

La Odontología es la rama de la Medicina que se encarga de diagnosticar y tratar diversas patologías que aquejan al sistema estomatognático; sin embargo, el odontólogo cirujano se va a ver enfrentado no solo a situaciones competentes a su área, sino también a las diversas condiciones sistémicas del paciente, la inyección de fármacos, el contacto con potenciales alérgenos como el látex y el uso de materiales dentales [8].

En Odontología, el estado de salud general del paciente es incierto, debido a que el paciente en la anamnesis no siempre es sincero por diferentes motivos; ya sea por descuido de salud, porque padece alguna enfermedad no diagnosticada o por vergüenza [9]. La gestión eficaz de una situación de emergencia en el consultorio dental es responsabilidad del odontólogo, quien debe ser capaz de diagnosticar los signos y los síntomas, y controlarlos adecuadamente. Si esto se logra, el profesional podrá brindar un tratamiento más completo $\mathrm{y}$ seguro [10-13].

La preparación del cirujano dentista ante las emergencias médicas viene relacionada con la capacidad de formación de los centros universitarios y con la actualización continua del profesional [14]. Existen pocos estudios que evalúan el nivel de formación de los estudiantes de Odontología en una emergencia médica. Además, las pocas investigaciones realizadas demuestran que dicho conocimiento es deficiente e insuficiente. Esto constituye un gran problema, ya que demuestra que el profesional no sabe cómo actuar frente a dichas situaciones de emergencia, lo que implica no solo graves consecuencias en la salud del paciente, sino también problemas legales para el odontólogo. Por lo tanto, es muy importante estimar el nivel de formación y el nivel de conocimiento sobre emergencias médicas. Esto es necesario para conocer sus áreas de debilidad y desarrollar estrategias que garantizarán que se beneficien al máximo [15].

Se hace imprescindible que el odontólogo tome medidas apropiadas para la prevención y el manejo de tales situaciones en el consultorio dental. La prevención exitosa depende de la evaluación de la historia clínica y la condición física del paciente, así como de las modificaciones apropiadas del tratamiento. Se han realizado estudios para evaluar la preparación de los odontólogos en el manejo de emergencias médicas en Kentucky y en Florida (Estados Unidos), en Australia y en Gran Bretaña. Hicieron hincapié en la necesidad de mejorar la capacitación, la organización de los consultorios y la preparación avanzada para el manejo de emergencias de los pacientes [16]. Un estudio de Nueva Zelanda mostró que más del 50\% de los odontólogos estaban insatisfechos con la enseñanza del pregrado en el manejo de emergencias médicas; y otro estudio mostró que los estudiantes de pregrado de Odontología brasileños estaban igualmente insatisfechos [17].

La literatura pone en evidencia un nivel de conocimiento insuficiente acerca del manejo de emergencias en la consulta odontológica. Esto fue motivo para determinar, en la presente investigación, el nivel de conocimiento de los estudiantes de quinto y sexto año de la Facultad de Odontología Pierre Fauchard en cuanto al manejo de situaciones de emergencia médico-odontológicas que se pueden presentar durante la consulta. Con esto, se busca contribuir al mejoramiento continuo en la formación académica de la casa de estudios, con base en la obtención de datos objetivos sobre el nivel de conocimiento de los estudiantes, y así implementar cursos de capacitación orientados al manejo de emergencias médicas en estudiantes de la Facultad de Odontología Pierre Fauchard.

\section{Objetivo general}

El objetivo general de este trabajo fue determinar el nivel de conocimiento de los alumnos de quinto y sexto año de la Facultad de Odontología Pierre Fauchard sobre el manejo de situaciones de emergencias médicas que se pueden presentar durante la consulta odontológica. 


\section{Materiales y métodos}

El diseño de este trabajo fue observacional, descriptivo, de corte transversal. La población enfocada estuvo conformada por 176 alumnos de quinto y sexto año de la Facultad de Odontología Pierre Fauchard, en septiembre del 2016, con el objetivo de evaluar el nivel de conocimiento sobre el manejo de situaciones de emergencias médicas que se pueden presentar durante la consulta. La muestra fue de 146 alumnos, el muestreo fue de tipo no probabilístico y representó el $82,9 \%$ de la población total.

Los criterios de inclusión para esta investigación fueron alumnos de quinto y sexto año sin distinción de género debidamente matriculados que aceptaron completar el cuestionario. Los criterios de exclusión fueron: alumnos de quinto y sexto año que estaban ausentes el día de la encuesta, alumnos que se negaron a realizar la encuesta y las encuestas realizadas de manera incompleta, alumnos de primer a cuarto año y docentes de la facultad. Por medio de una carta, se le solicitó a la decana de la Facultad de Odontología Pierre Fauchard su consentimiento para recoger los datos.

Para la recolección de datos, se aplicó una encuesta de veinticinco preguntas cerradas. El instrumento se diseñó con base en el diagnóstico de emergencias médicas, en el cual se evaluó el conocimiento con respecto a los principales signos y síntomas de las emergencias médicas más frecuentes y al conocimiento de primeros auxilios, del que se evaluó el conocimiento en RCP básica, manejo de síncope, crisis hipertensiva, crisis de hiperglicemia y de obstrucciones agudas de vía aérea y relacionadas con el manejo de sustancias farmacológicas para contrarrestar determinadas emergencias médicas. Las variables de interés fueron: edad, género y conocimiento. El nivel de conocimiento fue calculado teniendo en cuenta la escala del $60 \%$.

Los recursos utilizados en esta investigación fueron: un alumno calibrado para hacer el levantamiento de datos, y una vez realizada la encuesta, fueron seleccionados de acuerdo con los criterios de inclusión y exclusión. Todos los datos fueron cargados y analizados por pregunta con base en la escala del $60 \%$, en la que se consideró favorable un total de quince respuestas correctas. El análisis de respuestas se realizó en una planilla de Microsoft Excel diseñada para el estudio, para luego cargar y procesar con el programa spss para Windows 11.5 utilizando estadística descriptiva.

En este estudio, se tuvieron en cuenta los siguientes asuntos éticos: el respeto, ya que los datos se tomaron con consentimiento y se manejó con confidencialidad la identidad de los alumnos; la justicia, ya que todos los alumnos de quinto y sexto año tuvieron la oportunidad de participar en esta encuesta; y el beneficio, ya que contribuirá al mejoramiento continuo de la formación académica.

\section{Resultados}

En el siguiente apartado, se encuentran los resultados obtenidos con base en encuestas a alumnos de quinto y sexto año de la Facultad de Odontología Pierre Fauchard durante septiembre del 2016, en las cuales se evaluó el nivel de conocimiento sobre el manejo de situaciones de emergencia que se pueden presentar durante la consulta odontológica.

En una población de 176 alumnos, se excluyeron ocho cuestionarios, ya que no contaban con los datos completos para llevar a cabo la estadística; y veintidós alumnos no respondieron el cuestionario por estar ausentes el día de la encuesta o no querer completar el cuestionario, con lo que quedó una muestra de 146 alumnos. En datos generales fueron agrupados de acuerdo con la edad y el sexo. Estos resultados se exponen mediante tablas con su correspondiente descripción.

Tabla 1. Distribución de frecuencia de alumnos en relación con la edad. N: 146

\begin{tabular}{ccc}
\hline Edad & Frecuencia & Porcentaje \\
\hline 22 años & 26 & 17,8 \\
$\mathbf{2 3}$ años & $\mathbf{5 2}$ & $\mathbf{3 5 , 6}$ \\
24 años & 34 & 23,3 \\
25 años & 23 & 15,8 \\
26 años & 4 & 2,7 \\
27 años & 3 & 2,1 \\
28 años & 2 & 1,4 \\
30 años & 2 & 1,4 \\
\hline Total & 146 & 100,0 \\
\hline
\end{tabular}

Fuente: elaboración propia 
En la Tabla l, se observa que la mayoría de los alumnos estuvo en el rango de 23 años de edad con 52 encuestados, lo que representa el 35,6\%.

En la Tabla 2, se observa que el género con mayor número de alumnos es el femenino con 128 encuestados, lo que representa el $87,7 \%$.

Tabla 2. Distribución de frecuencia de alumnos en relación con el género. N: 146

\begin{tabular}{ccc}
\hline Género & Frecuencia & Porcentaje \\
\hline Masculino & 18 & 12,3 \\
Femenino & $\mathbf{1 2 8}$ & $\mathbf{8 7 , 7}$ \\
\hline Total & 146 & 100,0 \\
\hline
\end{tabular}

Fuente: elaboración propia

En la Tabla 3, se observa que los alumnos que respondieron "No" fueron mayoría con 120 encuestados, lo que representa el $82,2 \%$.

Tabla 3. Distribución de frecuencia de alumnos que han participado en cursos de primeros auxilios. N: 146

\begin{tabular}{ccc}
\hline \multicolumn{2}{c}{ ¿Ha participado usted en un curso de primeros auxilios? } \\
\hline Pregunta 1 & Frecuencia & Porcentaje \\
\hline Sí & 26 & 17,8 \\
No & $\mathbf{1 2 0}$ & $\mathbf{8 2 , 2}$ \\
\hline Total & 146 & 100,0 \\
\hline
\end{tabular}

Fuente: elaboración propia

En la Tabla 4, se observa que la cantidad de alumnos que participaron en el curso teórico-práctico fue de dieciséis, lo que representa el 11,0\%.

Tabla 4. Distribución de frecuencia de los alumnos con respuesta afirmativa a la pregunta 1 en relación con la participación en cursos teóricos o teórico-prácticos. N: 146

\begin{tabular}{ccc}
\hline \multicolumn{3}{c}{ En caso de responder $S i ́$, aclare si fue teórico o teórico práctico } \\
\hline Pregunta 1.1 & Frecuencia & Porcentaje \\
\hline Teórico & 10 & 6,8 \\
Teórico-practico & $\mathbf{1 6}$ & $\mathbf{1 1 , 0}$ \\
\hline Total & 146 & 100,0 \\
\hline
\end{tabular}

Fuente: elaboración propia
En la Tabla 5, se observa que respondieron correctamente 138 alumnos, lo que representa el $94,5 \%$.

Tabla 5. Distribución de frecuencia de respuestas correctas de los alumnos con respecto a qué es una situación de emergencia. $\mathrm{N}: 146$

\begin{tabular}{ccc}
\hline \multicolumn{3}{c}{ ¿Qué es una situación de emergencia para usted? } \\
\hline Pregunta 2 & Frecuencia & Porcentaje \\
\hline${ }^{*} \mathrm{~A}$ & 6 & $4,1 \%$ \\
${ }^{* *} \mathrm{~B}$ & 1 &, $7 \%$ \\
${ }^{* * *} \mathbf{C}$ & $\mathbf{1 3 8}$ & $\mathbf{9 4 , 5 \%}$ \\
${ }^{* * * *} \mathrm{D}$ & 1 &, $7 \%$ \\
\hline Total & 146 & $100,0 \%$ \\
\hline
\end{tabular}

*(A) Situación en la que el paciente manifiesta dolor en un diente después del tratamiento dental, ${ }^{* *}$ (в) Situación en la que el paciente tiene pocos recursos económicos para realizarse algún tratamiento dental, ${ }^{* * *}$ (c) Situación eventual crítica en la salud del paciente que tiene que ser socorrida de forma inmediata $y$ que compromete la vida del paciente, ${ }^{* * *}$ (D) Situación eventual semicrítica en la salud del paciente que tiene que ser socorrida pero puede ser durante el transcurso de los días.

Fuente: elaboración propia

En la Tabla 6, se observa que respondieron correctamente 86 alumnos, lo que representa el $58,9 \%$.

Tabla 6. Distribución de frecuencia de respuestas correctas de los alumnos con respecto a los signos clínicos del paro cardiorrespiratorio. N: 146

¿Qué emergencia médica presenta los signos clínicos de pérdida repentina de la conciencia, desaparición de los pulsos carotídeos y ruidos cardíacos, palidez mucocutánea intensa y cianosis?

\begin{tabular}{ccc}
\hline Pregunta 3 & Frecuencia & Porcentaje \\
\hline${ }^{*} \mathrm{~A}$ & 36 & $24,7 \%$ \\
${ }^{* *} \mathrm{~B}$ & 19 & $13,0 \%$ \\
${ }^{* * *} \mathrm{C}$ & 5 & $3,4 \%$ \\
${ }^{* * * *} \mathbf{D}$ & $\mathbf{8 6}$ & $\mathbf{5 8 , 9 \%}$ \\
\hline Total & 146 & $100,0 \%$ \\
\hline
\end{tabular}

${ }^{*}$ (A) Infarto agudo de miocardio, ${ }^{* *}$ (B) Anafilaxis, ${ }^{* * *}$ (C) Asma bronquial, ${ }^{* * * *}$ (D) Paro cardiorrespiratorio.

Fuente: elaboración propia 
En la Tabla 7, se observa que respondieron correctamente 101 alumnos, lo que representa el $69,2 \%$.

Tabla 7. Distribución de frecuencia de respuestas correctas de los alumnos con respecto a los signos clínicos del síncope vasovagal severo. N: 146

¿A qué emergencia médica le corresponden los signos clínicos de pérdida de conciencia, palidez, sudoración y descenso del pulso?

\begin{tabular}{ccc}
\hline Pregunta 4 & Frecuencia & Porcentaje \\
\hline${ }^{*} \mathrm{~A}$ & 16 & 11,0 \\
${ }^{* *} \mathbf{B}$ & 101 & $\mathbf{6 9 , 2}$ \\
${ }^{* * *} \mathrm{C}$ & 9 & 6,2 \\
${ }^{* * *} \mathrm{D}$ & 20 & 13,7 \\
\hline Total & 146 & 100,0 \\
\hline
\end{tabular}

${ }^{*}(\mathrm{~A})$ Infarto agudo de miocardio, ${ }^{* *}(\mathrm{~B})$ Síncope vasovagal severo, ${ }^{* * *}(\mathrm{C})$ Asma bronquial, ${ }^{* * * *}(\mathrm{D})$ Crisis hipertensiva.

Fuente: elaboración propia

En la Tabla 8, se puede observar que respondieron correctamente 61 alumnos, lo que representa el $41,8 \%$.

Tabla 8. Distribución de frecuencia de respuestas correctas de los alumnos con respecto a los signos clínicos del síndrome convulsivo. N: 146

\begin{tabular}{ccc}
\hline \multicolumn{3}{c}{$\begin{array}{c}\text { Cuáles son los principales signos clínicos para } \\
\text { diagnosticar un síndrome convulsivo? }\end{array}$} \\
\hline Pregunta 5 & Frecuencia & Porcentaje \\
\hline${ }^{*} \mathbf{A}$ & $\mathbf{6 1}$ & $\mathbf{4 1 , 8}$ \\
${ }^{* * \mathrm{~B}}$ & 24 & 16,4 \\
${ }^{* * *} \mathrm{C}$ & 44 & 30,1 \\
${ }^{* * *} \mathrm{D}$ & 17 & 11,6 \\
\hline Total & 146 & 100 \\
\hline
\end{tabular}

*(A) Presencia de contracciones tónicas o clónicas o tónico-clónicas de forma focal o generalizada, ${ }^{* *}$ (в) Cefalea, desorientación, pérdida de conciencia y contracciones clónicas, ${ }^{* * *}(\mathrm{C})$ Contracciones tónicas, desorientación, pérdida de conciencia y bradicardia, ${ }^{* * *}$ (D) Cefalea, inconsciencia progresiva, contracciones tónicas y sudoración.

Fuente: elaboración propia
En la Tabla 9, se puede observar que respondieron correctamente 48 alumnos, lo que representa el $32,9 \%$.

Tabla 9. Distribución de frecuencia de respuestas correctas de los alumnos con respecto a un cuadro de reacción tóxica al anestésico local. N: 146

\begin{tabular}{ccc}
\hline $\begin{array}{c}\text { Un cuadro de convulsiones, taquicardia, hipotensión, } \\
\text { aumento del ritmo respiratorio, náuseas y vómitos } \\
\text { puede presentarse cuando se trata de un(a): }\end{array}$ \\
\hline Pregunta 6 & Frecuencia & Porcentaje \\
\hline${ }^{*} \mathrm{~A}$ & 72 & 49,3 \\
${ }^{* *} \mathrm{~B}$ & 17 & 11,6 \\
${ }^{* * *} \mathrm{C}$ & $\mathbf{4 8}$ & $\mathbf{3 2 , 9}$ \\
${ }^{* * * *} \mathrm{D}$ & 9 & 6,2 \\
\hline Total & 146 & 100,0 \\
\hline
\end{tabular}

${ }^{*}(\mathrm{~A})$ Shock anafiláctico, ${ }^{* *}(\mathrm{~B})$ Crisis hipertensiva, ${ }^{* * *}(\mathrm{C})$ Reac-

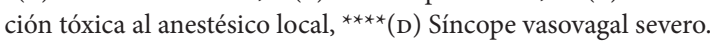
Fuente: elaboración propia

En la Tabla 10, se observa que respondieron correctamente ochenta alumnos, lo que representa el $54,8 \%$.

Tabla 10. Distribución de frecuencia de respuestas correctas de los alumnos con respecto a un cuadro de anafilaxis severa. $\mathrm{N}: 146$

\begin{tabular}{ccc}
\hline $\begin{array}{c}\text { ¿Qué signos clínicos en un paciente pueden orientar } \\
\text { nuestro diagnóstico hacia un cuadro de anafilaxis } \\
\text { severa durante la consulta odontológica? }\end{array}$ \\
\hline Pregunta 7 & Frecuencia & Porcentaje \\
\hline${ }^{*} \mathrm{~A}$ & 12 & 8,2 \\
${ }^{* *} \mathbf{B}$ & $\mathbf{8 0}$ & $\mathbf{5 4 , 8}$ \\
${ }^{* * *} \mathrm{C}$ & 23 & 15,8 \\
${ }^{* * * *} \mathrm{D}$ & 31 & 21,2 \\
\hline Total & 146 & 100,0 \\
\hline
\end{tabular}

*(A) Presencia de contracciones tónico-clónicas, hipotensión severa y repentina pérdida de la conciencia, ${ }^{* * *}$ (B) Urticaria generalizada, palidez, frialdad, sudoración, broncoespasmo, posterior pérdida de conciencia e hipotensión severa, ${ }^{* * *}$ (c) Presencia de convulsiones, sudoración profusa, taquicardia, bochornos y aumento del ritmo respiratorio, ${ }^{* * *}$ (D) Presencia de palidez cutánea, broncoespasmo y desaparición de pulsos carotídeos.

Fuente: elaboración propia 
En la Tabla 11, se observa que respondieron correctamente 85 alumnos, lo que representa el $58,2 \%$.

Tabla 11. Distribución de frecuencia de respuestas correctas de los alumnos con respecto al diagnóstico de una crisis hipertensiva. N: 146

\begin{tabular}{|c|c|c|}
\hline \multicolumn{3}{|c|}{$\begin{array}{c}\text { Cuando un paciente atraviesa un cuadro de presión } \\
\text { arterial diastólica mayor a } 120 \mathrm{~mm} \text { Hg, cefalea, lesiones } \\
\text { oculares (hemorragia, exudados), hematuria y convulsiones, } \\
\text { se puede diagnosticar un(a): }\end{array}$} \\
\hline Pregunta 8 & Frecuencia & Porcentaje \\
\hline${ }^{*} \mathrm{~A}$ & 29 & $19,9 \%$ \\
\hline${ }^{* *} \mathrm{~B}$ & 22 & $15,1 \%$ \\
\hline${ }^{* * *} \mathrm{C}$ & 10 & $6,8 \%$ \\
\hline${ }^{* * * *} \mathbf{D}$ & 85 & $58,2 \%$ \\
\hline Total & 146 & $100,0 \%$ \\
\hline
\end{tabular}

${ }^{*}$ (А) Crisis de hiperglicemia, ${ }^{* * *}$ (в) Infarto agudo de miocardio, ${ }^{* * *}$ (C) Shock anafiláctico, ${ }^{* * * *}$ (D) Crisis hipertensiva.

Fuente: elaboración propia

En la Tabla 12, se puede observar que respondieron correctamente 119 alumnos, lo que representa el $81,5 \%$.

Tabla 12. Distribución de frecuencia de respuestas correctas de los alumnos con respecto a los signos clínicos de crisis de hiperglicemia. N: 146

Los signos clínicos de presencia de fuerte dolor abdominal, vómitos, taquipnea, y cuando en el glucómetro se registran valores mayores a $200 \mathrm{mg} / \mathrm{dL}$ de glucosa en sangre podrían tratarse de un(a):

\begin{tabular}{ccc}
\hline Pregunta 9 & Frecuencia & Porcentaje \\
\hline${ }^{*} \mathrm{~A}$ & $\mathbf{1 1 9}$ & $\mathbf{8 1 , 5 \%}$ \\
${ }^{*{ }_{\mathrm{B}}}$ & 8 & $5,5 \%$ \\
${ }^{* * *} \mathrm{C}$ & 9 & $6,2 \%$ \\
${ }^{* * * *} \mathrm{D}$ & 10 & $6,8 \%$ \\
\hline Total & 146 & $100,0 \%$ \\
\hline
\end{tabular}

${ }^{*}$ (А) Crisis de hiperglicemia, ${ }^{* * *}$ (в) Crisis asmática aguda, ${ }^{* * *}$ (C) Shock anafiláctico, ${ }^{* * * *}$ (D) Paro cardiorrespiratorio. Fuente: elaboración propia
En la Tabla 13, se observa que respondieron correctamente 69 alumnos, lo que representa el $47,3 \%$.

Tabla 13. Distribución de frecuencia de respuestas correctas de los alumnos con respecto al diagnóstico de crisis aguda de asma bronquial. $\mathrm{N}: 146$

Cuando el cuadro inicia con tos productiva acompañada de broncoespasmo, intensa disnea, retracciones de los músculos intercostales (tiraje) y cianosis de la mucosa labial $\mathrm{y}$ base de las uñas, puede diagnosticarse un(a):

\begin{tabular}{ccc}
\hline Pregunta $\mathbf{1 0}$ & Frecuencia & Porcentaje \\
\hline${ }^{*} \mathrm{~A}$ & 6 & 4,1 \\
${ }^{* *} \mathrm{~B}$ & 15 & 10,3 \\
${ }^{* * *} \mathrm{C}$ & $\mathbf{6 9}$ & $\mathbf{4 7 , 3}$ \\
${ }^{* * *} \mathrm{D}$ & 56 & 38,4 \\
\hline Total & 146 & 100,0 \\
\hline
\end{tabular}

${ }^{*}$ (A) Shock anafiláctico, ${ }^{* * *}$ (B) Paro cardiorrespiratorio, ${ }^{* * *}$ (C) Crisis aguda de asma bronquial, ${ }^{* * * *}(\mathrm{D})$ Obstrucción aguda de vías respiratorias.

Fuente: elaboración propia

En la Tabla 14, se observa que respondieron correctamente 85 alumnos, lo que representa el $58,2 \%$.

Tabla 14. Distribución de frecuencia de respuestas correctas de los alumnos con respecto a signos clínicos de una obstrucción aguda de vías respiratorias. N: 146

¿Qué signos clínicos en un paciente pueden orientar nuestro diagnóstico hacia una obstrucción aguda de vías respiratorias durante la consulta odontológica?

\begin{tabular}{ccc}
\hline Pregunta 11 & Frecuencia & Porcentaje \\
\hline${ }^{*}$ A & 34 & 23,3 \\
${ }^{* *}$ B & $\mathbf{8 5}$ & $\mathbf{5 8 , 2}$ \\
${ }^{* * *} \mathrm{C}$ & 15 & 10,3 \\
${ }^{* * * *} \mathrm{D}$ & 12 & 8,2 \\
\hline Total & 146 & 100,0 \\
\hline
\end{tabular}

${ }^{*}$ (A) Presencia de broncoespasmo, hipotensión severa y repentina pérdida de la conciencia, ${ }^{* * *}$ (в) Presencia de estridor, alteraciones o ausencia de la fonación, tiraje, "ahogo" o asfixia, ${ }^{* * *}$ (C) Presencia de convulsiones, sudoración, taquicardia y aumento del ritmo respiratorio, ${ }^{* * *}$ (D) Presencia de estridor, tiraje, afonía y desaparición de pulsos carotídeos.

Fuente: elaboración propia 
En la Tabla 15, se observa que respondieron correctamente 54 alumnos, lo que representa el $37,0 \%$.

Tabla 15. Distribución de frecuencia de respuestas correctas de los alumnos con respecto a primeros auxilios del síncope. N: 146

\begin{tabular}{ccc}
\hline $\begin{array}{c}\text { Si se presentara un síncope vasovagal al paciente, lo } \\
\text { recomendable es colocarlo en la posición de Tren de Lemburg } \\
\text { o posición antishock. ¿En qué consiste esta posición? }\end{array}$ \\
\hline Pregunta $\mathbf{1 2}$ & Frecuencia & Porcentaje \\
\hline${ }^{*} \mathrm{~A}$ & 45 & 30,8 \\
${ }^{* *} \mathrm{~B}$ & 28 & 19,2 \\
${ }^{* * *} \mathrm{C}$ & 19 & 13,0 \\
${ }^{* * * *} \mathbf{D}$ & $\mathbf{5 4}$ & $\mathbf{3 7 , 0}$ \\
\hline Total & 146 & 100,0 \\
\hline
\end{tabular}

${ }^{*}$ (A) Poner al paciente con la espalda a $45^{\circ}$ supina, y con elevación de los miembros inferiores, ${ }^{* * *}$ (в) Poner al paciente en posición horizontal, cúbito ventral y con elevación de piernas, ${ }_{* * *}(\mathrm{C})$ Poner al paciente en posición de cúbito dorsal, con elevación de brazos y cabeza, ${ }^{* * * *}$ (D) Poner al paciente en posición horizontal, supina, con elevación de los miembros inferiores. Fuente: elaboración propia

En la Tabla 16, se observa que respondieron correctamente 56 alumnos, lo que representa el $38,4 \%$.

Tabla 16. Distribución de frecuencia de respuestas correctas de los alumnos con respecto a la conducta terapéutica a seguir en emergencias hipertensivas. N: 146

En cuadros de emergencias hipertensivas, ¿cuál es la conducta terapéutica que debemos seguir?

\begin{tabular}{ccc}
\hline Pregunta 13 & Frecuencia & Porcentaje \\
\hline${ }^{*} \mathrm{~A}$ & 18 & 12,3 \\
${ }^{* *} \mathrm{~B}$ & 36 & 24,7 \\
${ }^{* *} \mathrm{C}$ & $\mathbf{5 6}$ & $\mathbf{3 8 , 4}$ \\
${ }^{* * * *} \mathrm{D}$ & 36 & 24,7 \\
\hline Total & 146 & 100 \\
\hline
\end{tabular}

*(A) Proporcionar una toma de ácido acetilsalicílico de $100 \mathrm{mg}$ y esperar media hora, ${ }^{* *}($ в) Proporcionar adrenalina 0,1 a $0,5 \mathrm{~mL}$, luego brindar oxigenoterapia, ${ }^{\star * *}$ (C) Realizar soporte básico de vida si requiriese y trasladar rápido al paciente a un centro hospitalario, ${ }^{\star * * *}$ (D) Poner al paciente en posición de Tren de Lemburg y proporcionar ventilación constante.

Fuente: elaboración propia
En la Tabla 17, se observa que respondieron correctamente 59 alumnos, lo que representa el $40,4 \%$.

Tabla 17. Distribución de frecuencia de respuestas correctas de los alumnos con respecto a los valores normales del tiempo de coagulación y tiempo de sangría. N: 146

Con respecto a las pruebas de laboratorio, ¿cuáles son los valores normales del tiempo de coagulación y tiempo de sangría?

\begin{tabular}{ccc}
\hline Pregunta 14 & Frecuencia & Porcentaje \\
\hline${ }^{*}$ A & 43 & 29,5 \\
${ }^{* * *}$ B & 22 & 15,1 \\
${ }^{* * *}$ C & $\mathbf{5 9}$ & $\mathbf{4 0 , 4}$ \\
${ }^{* * * *} \mathrm{D}$ & 22 & 15,1 \\
\hline Total & 146 & 100 \\
\hline
\end{tabular}

*(A) Mayor o igual a 11 minutos y menor o igual a 6 minutos. **(в) Menor o igual a 15 minutos y menor o igual a 8 minutos. $* * *$ (C) Menor o igual a 11 minutos y menor o igual a 6 minutos. $* * * *$ (D) Menor o igual a 4 minutos y menor o igual a 3 minutos. Fuente: elaboración propia

En la Tabla 18, se observa que respondieron correctamente 66 alumnos, lo que representa el $45,2 \%$.

Tabla 18. Distribución de frecuencia de respuestas correctas de los alumnos con respecto al manejo de un paciente con hiperventilación. N: 146

El síndrome de hiperventilación aparece cuando el
paciente ansioso empieza a respirar rápidamente,
eliminando anormalmente el co 2 . En este caso usted:

\begin{tabular}{ccc}
\hline Pregunta 15 & Frecuencia & Porcentaje \\
\hline${ }^{*} \mathrm{~A}$ & 8 & $5,5 \%$ \\
${ }^{* * *} \mathrm{~B}$ & 39 & $26,7 \%$ \\
${ }^{* * *} \mathrm{C}$ & 33 & $22,6 \%$ \\
${ }^{* * * *} \mathbf{D}$ & $\mathbf{6 6}$ & $\mathbf{4 5 , 2 \%}$ \\
\hline Total & 146 & $100 \%$ \\
\hline
\end{tabular}

*(A) Tranquiliza al paciente verbalmente para que se calme y lo manda para la casa, ${ }^{* *}($ в) Le da oxígeno 1 a $3 \mathrm{~L} / \mathrm{min}$, lo tranquiliza verbalmente y continúa con el tratamiento dental, ${ }^{* * *}$ (c) Le da diazepam en ampolla de $0,15 \mathrm{mg} / \mathrm{kg}$ Iv, lo tranquiliza verbalmente y continúa con el tratamiento dental, ${ }^{* * *}$ (D) Tranquiliza verbalmente al paciente, le da respiración guiada (técnica de respiración dentro de una bolsa), no usa oxígeno, continúa con el tratamiento dental.

Fuente: elaboración propia 
En la Tabla 19, se observa que respondieron correctamente 76 alumnos, lo que representa el $52,1 \%$.

Tabla 19. Distribución de frecuencia de respuestas correctas de los alumnos con respecto al manejo de un paciente con obstrucción de las vías aéreas. N: 146

\begin{tabular}{ccc}
\hline \multicolumn{2}{c}{ ¿Qué medidas tomaría si el paciente sufre una } \\
obstrucción aguda de vías aéreas en su consulta?
\end{tabular}

*(A) Realizar RCP básico, extenderle los brazos y acostar al paciente, ${ }^{* *}$ (в) Se pone al paciente cabeza abajo, se ayuda de palmadas en la espalda para que expulse el objeto; si el cuadro es grave, realizar traqueotomía, ${ }^{* * *}$ (c) Abrazar al paciente desde atrás, presionando el epigastrio para aumentar la presión intratorácica y que expulse el objeto (maniobra de Heimlich); en casos muy graves, realizar cricotiroidotomía, ${ }^{* * *}$ (D) Se pone al paciente boca abajo, se le dan palmadas en la espalda y luego se introduce el dedo en la boca para intentar sacar el cuerpo extraño.

Fuente: elaboración propia

En la Tabla 20, se observa que respondieron correctamente 79 alumnos, lo que representa el $54,1 \%$.

Tabla 20. Distribución de frecuencia de respuestas correctas de los alumnos con respecto al manejo de un paciente con cuadro de hiperglicemia. N: 146

¿Cómo contrarrestaría cuadros críticos de hiperglicemia de un paciente en la consulta?

\begin{tabular}{ccc}
\hline Pregunta 17 & Frecuencia & Porcentaje \\
\hline${ }^{*} \mathbf{A}$ & $\mathbf{7 9}$ & $\mathbf{5 4 , 1}$ \\
${ }^{* *} \mathrm{~B}$ & 37 & 25,3 \\
${ }^{* * *} \mathrm{C}$ & 7 & 4,8 \\
${ }^{* * * *} \mathrm{D}$ & 23 & 15,8 \\
\hline Total & 146 & 100 \\
\hline
\end{tabular}

${ }^{*}$ (A) Insulina SC, ${ }^{* *}$ (в) Glibenclamida $5 \mathrm{mg}+$ suero salino hipotónico, ${ }^{* * *}$ (C) Diazepam $10 \mathrm{mg}$ IM, ${ }^{* * * *}$ (D) Metformina $850 \mathrm{mg}$ vo + suero salino hipotónico.

Fuente: elaboración propia
En la Tabla 21, se observa que respondieron correctamente 33 alumnos, lo que representa el $22,6 \%$.

Tabla 21. Distribución de frecuencia de respuestas correctas de los alumnos con respecto al manejo de un paciente con cuadro de convulsión. N: 146

¿Qué conducta adoptaría si su paciente empieza a padecer un síndrome convulsivo en su atención odontológica?

\begin{tabular}{ccc}
\hline Pregunta $\mathbf{1 8}$ & Frecuencia & Porcentaje \\
\hline${ }^{*} \mathrm{~A}$ & 41 & 28,1 \\
${ }^{* *} \mathrm{~B}$ & 39 & 26,7 \\
${ }^{* * *} \mathrm{C}$ & $\mathbf{3 3}$ & $\mathbf{2 2 , 6}$ \\
${ }^{* * * *} \mathrm{D}$ & 33 & 22,6 \\
\hline Total & 146 & 100 \\
\hline
\end{tabular}

*(A) Esperar a que la reacción pase y llevarlo lo antes posible a un centro médico, ${ }^{* *}(\mathrm{~B})$ Proteger de traumatismos al paciente y aplicarle diazepam $10 \mathrm{mg}$ IM o $0,15 \mathrm{mg} / \mathrm{kg} /$ dosis diluido en $10 \mathrm{~cm}^{3}$ de dextrosa al $5 \%,{ }^{* * *}$ (c) Mantener las vías permeables, prevenir la broncoaspiración elevando la cabeza del paciente $20^{\circ}$, lateralizar la cabeza y aplicarle midazolam $5 \mathrm{mg}$ Ev en 10 $\mathrm{cm}^{3}$ diluido en solución salina, ${ }^{* * * *}$ (D) Sujetar con fuerza sus extremidades y cabeza y llamar a un médico.

Fuente: elaboración propia

En la Tabla 22, se observa que respondieron correctamente 46 alumnos, lo que representa el $31,5 \%$.

Tabla 22. Distribución de frecuencia de respuestas correctas de los alumnos con respecto al manejo del medicamento utilizado en un paciente con cuadro de convulsión. N: 146

En una situación grave de intoxicación por anestésico local, ¿qué tipo de medicamento le administraría a su paciente para contrarrestar su principal signo clínico (convulsión)?

\begin{tabular}{ccc}
\hline Pregunta 19 & Frecuencia & Porcentaje \\
\hline${ }^{*} \mathrm{~A}$ & 9 & 6,2 \\
${ }^{* *} \mathrm{~B}$ & 23 & 15,8 \\
${ }^{* * *} \mathrm{C}$ & 68 & 46,6 \\
${ }^{* * * *} \mathbf{D}$ & $\mathbf{4 6}$ & $\mathbf{3 1 , 5}$ \\
\hline Total & 146 & 100 \\
\hline
\end{tabular}

${ }^{*}$ (A) Aspirina $100 \mathrm{mg}$ vo, ${ }^{* *}$ (B) Bisoprolol $10 \mathrm{mg}$ vo, ${ }^{* * *}$ (C) Nitroglicerina $0,5 \mathrm{mg} \mathrm{SL},{ }^{* * * *}$ (D) Diazepam $10 \mathrm{mg}$ EV. Fuente: elaboración propia 
En la Tabla 23, se observa que respondieron correctamente 48 alumnos, lo que representa el $32,9 \%$.

Tabla 23. Distribución de frecuencia de respuestas correctas de los alumnos con respecto al manejo farmacológico en un paciente con anafilaxis. N: 146

\begin{tabular}{ccc}
\hline \multicolumn{3}{c}{ ¿Qué medidas tomaría ante una situación } \\
de anafilaxis en su paciente? \\
\hline Pregunta 20 & Frecuencia & Porcentaje \\
\hline${ }^{*} \mathrm{~A}$ & 34 & 23,3 \\
${ }^{* *} \mathrm{~B}$ & 48 & 32,9 \\
${ }^{* * *} \mathbf{C}$ & $\mathbf{4 8}$ & $\mathbf{3 2 , 9}$ \\
${ }^{* * *} \mathrm{D}$ & 16 & 11,0 \\
\hline Total & 146 & 100 \\
\hline
\end{tabular}

*(A) Ventilación con intubación, posición horizontal y solo aplicación de dexametasona IM $4 \mathrm{mg},{ }^{* *}($ B) Posición de Tren de Lemburg, RCP, aplicación de un antihistamínico y diazepam 5 mg Ev, ${ }^{* * *}(\mathrm{c})$ Posición de Tren de Lemburg, intubación si requiriese, aplicación de epinefrina sc 0,2 a $0,5 \mathrm{~mL}$; luego dexametasona $4 \mathrm{mg}$ y un antihistamínico, ${ }^{* * *}$ (D) Posición cúbito dorsal, ventilación manual y administración de cloranfenicol Ev.

Fuente: elaboración propia

En la Tabla 24, se puede observar que respondieron correctamente 76 alumnos, lo que representa el $52,1 \%$.

Tabla 24. Distribución de frecuencia de respuestas correctas de los alumnos con respecto al manejo farmacológico en un paciente con crisis asmática. N: 146

\begin{tabular}{ccc}
\hline \multicolumn{3}{c}{$\begin{array}{c}\text { ¿Qué tipo de fármaco le administraría a su paciente } \\
\text { si se le presenta una crisis asmática aguda durante } \\
\text { su atención en el consultorio dental? }\end{array}$} \\
\hline Pregunta 21 & Frecuencia & Porcentaje \\
\hline${ }^{*} \mathbf{A}$ & $\mathbf{7 6}$ & $\mathbf{5 2 , 1}$ \\
${ }^{* *} \mathrm{~B}$ & 9 & 6,2 \\
${ }^{* * *} \mathrm{C}$ & 15 & 10,3 \\
${ }^{* * * *} \mathrm{D}$ & 46 & 31,5 \\
\hline Total & 146 & 100 \\
\hline
\end{tabular}

${ }^{*}$ (A) Salbutamol $100 \mathrm{mcg} / 2$ puff $\mathrm{v}$ inhalatoria, ${ }^{* *}$ (в) Midazolam $5 \mathrm{mg}$ vo, ${ }^{* *}$ (C) Captopril $25 \mathrm{mg} \mathrm{sL},{ }^{* * *}$ (D) Oxígeno $100 \mathrm{~mL} \mathrm{v}$ inhalatoria.

Fuente: elaboración propia
En la Tabla 25, se observa que respondieron correctamente 34 alumnos, lo que representa el $23,3 \%$.

Tabla 25. Distribución de frecuencia de respuestas correctas de los alumnos con respecto a los valores normales de la frecuencia respiratoria. N: 146

¿Cuál de los siguientes son los valores normales de la frecuencia respiratoria en un paciente sano?

\begin{tabular}{ccc}
\hline Pregunta 22 & Frecuencia & Porcentaje \\
\hline${ }^{*} \mathrm{~A}$ & 77 & 52,7 \\
${ }^{* *} \mathbf{B}$ & $\mathbf{3 4}$ & $\mathbf{2 3 , 3}$ \\
${ }^{* * *} \mathrm{C}$ & 19 & 13,0 \\
${ }^{* * * *} \mathrm{D}$ & 16 & 11,0 \\
\hline Total & 146 & 100 \\
\hline
\end{tabular}

${ }^{*}$ (A) 70-90/mnt, ${ }^{* *}$ (B) $12-20 / \mathrm{mnt},{ }^{* * *}$ (C) $120-60 / \mathrm{mnt},{ }^{* * *}$ (D) 30-60/mnt.

Fuente: elaboración propia

En la Tabla 26, se observa que respondieron correctamente 45 alumnos, lo que representa el $30,8 \%$.

Tabla 26. Distribución de frecuencia de respuestas correctas de los alumnos con respecto al manejo en un paciente que aspira un cuerpo extraño. N: 146

Si un paciente aspira un cuerpo extraño mientras se encuentra sentado en la silla dental y empieza a toser y a manifestar dificultad respiratoria, ¿cuáles serían los pasos a seguir para tratar esta emergencia?:

\begin{tabular}{ccc}
\hline Pregunta 23 & Frecuencia & Porcentaje \\
\hline${ }^{*} \mathrm{~A}$ & 32 & $21,9 \%$ \\
${ }^{* *} \mathrm{~B}$ & 33 & $22,6 \%$ \\
${ }^{* * *} \mathrm{C}$ & 36 & $24,7 \%$ \\
${ }^{* * *} \mathbf{D}$ & $\mathbf{4 5}$ & $\mathbf{3 0 , 8 \%}$ \\
\hline Total & 146 & $100 \%$ \\
\hline
\end{tabular}

*(A) Llamar a la línea de emergencias, poner al paciente inclinado hacia adelante con la cabeza entre las rodillas y pedirle que tosa hasta que salga el objeto, darle oxígeno, ${ }^{* *}$ (в) Suministrarle oxígeno, tenerlo bajo vigilancia continua y enviarlo a un hospital, ${ }^{* * *}$ (c) Realizarle el RCP, llamar a la línea de emergencias y revisar los signos vitales cada cinco minutos mientras viene la ayuda profesional, ${ }^{* * *}$ (D) Llamar a la línea de emergencias, preguntarle al paciente si se está atragantando, pedirle al paciente que tosa y si el objeto no sale, empezar la maniobra de Heimlich.

Fuente: elaboración propia 
En la Tabla 27, se observa que respondieron correctamente 85 alumnos, lo que representa el $58,2 \%$.

Tabla 27. Distribución de frecuencia de respuestas correctas de los alumnos con respecto al diagnóstico de una crisis hipertensiva. N: 146

\begin{tabular}{|c|c|c|}
\hline \multicolumn{3}{|c|}{$\begin{array}{l}\text { Si mientras esta en la sala de espera del consultorio un } \\
\text { paciente empieza a manifestar taquicardia, mareos, } \\
\text { palpitaciones, malestar general, opresión torácica, presión } \\
\text { arterial de } 150 / 110 \mathrm{Hg} \text {, se dice que se está ante la presencia de }\end{array}$} \\
\hline Pregunta 24 & Frecuencia & Porcentaje \\
\hline${ }^{*} \mathrm{~A}$ & 15 & 10,3 \\
\hline${ }^{* *} \mathbf{B}$ & 85 & 58,2 \\
\hline${ }^{* * *} \mathrm{C}$ & 15 & 10,3 \\
\hline${ }^{* * * *} \mathrm{D}$ & 31 & 21,2 \\
\hline Total & 146 & 100 \\
\hline
\end{tabular}

${ }^{*}$ (A) Una crisis de hiperventilación, ${ }^{* *}$ (B) Una crisis hipertensiva, ${ }^{* * *}$ (C) Una hiperglucemia, ${ }^{* * *}$ (D) Una crisis nerviosa.

Fuente: elaboración propia

En la Tabla 28, se observa que respondieron correctamente 85 alumnos, lo que representa el $58,2 \%$.

Tabla 28. Distribución de frecuencia de respuestas correctas de los alumnos con respecto a los signos y síntomas de un paciente con hemorragia. N: 146

\begin{tabular}{|c|c|c|}
\hline \multicolumn{3}{|c|}{$\begin{array}{l}\text { En un cuadro de hemorragia bucal, ¿qué signos y síntomas nos } \\
\text { darán referencia de que un paciente adulto perdió un volumen } \\
\text { sanguíneo de un aprox. } 650 \mathrm{cc} \text { a } 1000 \mathrm{cc} \text { en una cirugía bucal? }\end{array}$} \\
\hline Pregunta 25 & Frecuencia & Porcentaje \\
\hline${ }^{*} \mathrm{~A}$ & 11 & 7,5 \\
\hline${ }^{* *} \mathbf{B}$ & 64 & 43,8 \\
\hline${ }^{* * *} \mathrm{C}$ & 29 & 19,9 \\
\hline${ }^{* * * *} \mathrm{D}$ & 42 & 28,8 \\
\hline Total & 146 & 100 \\
\hline
\end{tabular}

${ }^{*}$ (А) Taquicardia-mareo-prurito-broncoespasmo, ${ }^{* *}($ в $)$ Taquicardia-mareo-hipotensión, ${ }^{* * *}$ (C) Mareo-prurito-hipotensión-broncoespasmo, ${ }^{* * *}(\mathrm{D})$ Taquicardia-mareo-hipotensión-broncoespasmo.

Fuente: elaboración propia
En la Tabla 29, se observan catorce respuestas desfavorables, lo que representa el 56\%, y once respuestas favorables, lo que representa el $44 \%$

Tabla 29. Distribución de frecuencia de respuestas Favorable y Desfavorable por preguntas N: 25

\begin{tabular}{|c|c|c|}
\hline Pregunta & \multicolumn{2}{|c|}{ Desfavorable / favorable } \\
\hline 1 & \multicolumn{2}{|r|}{ Desfavorable } \\
\hline 2 & \multicolumn{2}{|r|}{ Favorable } \\
\hline 3 & \multicolumn{2}{|r|}{ Favorable } \\
\hline 4 & \multicolumn{2}{|r|}{ Favorable } \\
\hline 5 & \multicolumn{2}{|r|}{ Desfavorable } \\
\hline 6 & \multicolumn{2}{|r|}{ Desfavorable } \\
\hline 7 & \multicolumn{2}{|r|}{ Favorable } \\
\hline 8 & \multicolumn{2}{|r|}{ Favorable } \\
\hline 9 & \multicolumn{2}{|r|}{ Favorable } \\
\hline 10 & \multicolumn{2}{|r|}{ Desfavorable } \\
\hline 11 & \multicolumn{2}{|r|}{ Favorable } \\
\hline 12 & \multicolumn{2}{|r|}{ Desfavorable } \\
\hline 13 & \multicolumn{2}{|r|}{ Desfavorable } \\
\hline 14 & \multicolumn{2}{|r|}{ Desfavorable } \\
\hline 15 & \multicolumn{2}{|r|}{ Desfavorable } \\
\hline 16 & \multicolumn{2}{|r|}{ Favorable } \\
\hline 17 & \multicolumn{2}{|r|}{ Favorable } \\
\hline 18 & \multicolumn{2}{|r|}{ Desfavorable } \\
\hline 19 & \multicolumn{2}{|r|}{ Desfavorable } \\
\hline 20 & \multicolumn{2}{|r|}{ Desfavorable } \\
\hline 21 & \multicolumn{2}{|r|}{ Favorable } \\
\hline 22 & \multicolumn{2}{|r|}{ Desfavorable } \\
\hline 23 & \multicolumn{2}{|r|}{ Desfavorable } \\
\hline 24 & \multicolumn{2}{|r|}{ Favorable } \\
\hline 25 & \multicolumn{2}{|r|}{ Desfavorable } \\
\hline Conocimiento & Frecuencia & $\%$ \\
\hline Desfavorable & 14 & $56 \%$ \\
\hline Favorable & 11 & $44 \%$ \\
\hline Total & 25 & $100 \%$ \\
\hline
\end{tabular}

Fuente: elaboración propia

Es desfavorable en un 56\% el nivel de conocimiento de los alumnos de quinto y sexto año acerca del manejo de situaciones de emergencia que se pueden presentar durante la consulta. 


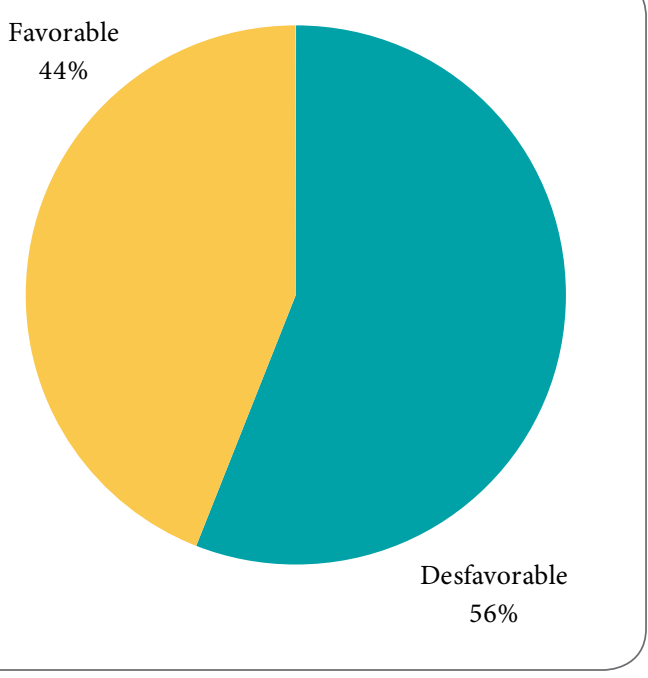

Figura 1. Distribución de frecuencia de respuestas Favorable y Desfavorable por preguntas. N: 25 Fuente: elaboración propia

\section{Discusión}

En esta investigación, se evaluó el nivel de conocimiento de 146 alumnos de quinto y sexto año de la Facultad de Odontología Pierre Fauchard sobre el manejo de situaciones de emergencias médicas. Se coincidió con Villena [18], de la ciudad de Trujillo, Perú, en cuanto a la población enfocada y al material y los métodos, que fue de tipo observacional descriptivo de corte transversal.

En cuanto a los resultados en este trabajo, se presenta un nivel desfavorable de conocimiento en el $56 \%$ de los alumnos, similar a lo hallado por Villena [18] (65,3\%), por Mckenzie [20] $(57,14 \%)$ y por Elanchezhiyan [14], Ehigiator [15] y Tunupriya y Aradhya [16], que fue bajo; y a diferencia de lo hallado por Contreras [2], que obtuvo un nivel regular con un porcentaje de $42,9 \%$, que coincidió con Torres [10] $(43,88 \%)$ y Hernández y Zapata [5] y Tupiza Guerrero [27] (80\%).

En estudios realizados a odontólogos en Perú por Gaviño y Rodrich [8], en el 2015, respecto al nivel de conocimiento de odontólogos sobre el manejo de emergencias durante la consulta odontológica, hubo un resultado regular con un porcentaje de 43,98\%, lo que coincidió con Jodalli y Ankola [21], y a diferencia de Benavides [14], Stafuzza et al. [4] y Arsati et al. [22], que tuvieron como resultado un nivel de conocimiento malo. En cambio, Bilich et al. [29] realizaron estudios en higienistas dentales, con una buena tasa de respuesta de más del $80 \%$. Y Mejía et al. [1] realizaron un estudio a estudiantes de Medicina en universidades peruanas en el 2011, el cual dio como resultado que el nivel de conocimiento no es bueno.

En relación con el género, en los resultados obtenidos en esta investigación hubo un porcentaje elevado del sexo femenino, de $87,7 \%$; semejante a lo hallado por Contreras [2] (44,9\%), Torres [10] y Gasco [6] (68,8\%), Benavides [14], Stafuzza [4] y Shenoy et al. [23].

Con respecto a los resultados de la participación en cursos de primeros auxilios, el 82,2\% de los alumnos (120) no ha participado en ningún curso; semejante a lo hallado por Contreras [2] $(83,7 \%)$ y Mejía (52,5\%) [1]. A diferencia de la investigación realizada por Gasco [6], en España el 100\% de los estudiantes recibió capacitación en RCP. En cuanto a la capacitación de odontólogos, Benavides [14] tuvo como resultado que el $85 \%$ ha recibido algún tipo de capacitación; semejante a lo hallado en la investigación realizada por Muller y Sther [24] (92\%), la de Arsati et al. [22] (59,6\%) y la de Shenoy [23] $50,70 \%$.

Con respecto a los alumnos que respondieron que sí, veintiséis participaron en cursos de primeros auxilios, diecisiéis participaron en cursos teórico-prácticos (11\%) y diez participaron en cursos teóricos $(6,8 \%)$. Semejante a lo hallado por Ehigiator [15] en su investigación a 124 estudiantes de Odontología en Nigeria en el 2014, veintisiete $(21,8 \%)$ han recibido capacitación teórica y $35(28,2 \%)$ han recibido capacitación teórico-práctica. A diferencia de Gasco [6], en el estudio realizado en España el 100\% contestó que había recibido enseñanza de RCP básica teórica y práctica. En cambio, Mutzbauer et al. [30], de Australia, realizaron una simulación incorporando un tutorial interactivo y escenarios de emergencia realistas simulados en el ambiente clínico real de los estudiantes de la Universidad de Melbourne, con lo que concluyeron que es un complemento eficaz a la enseñanza tradicional.

Con respecto a la definición de emergencia, 138 alumnos $(94,5 \%)$ de quinto y sexto año respondieron correctamente, a diferencia del estudio de Contreras [2], realizado en Perú a alumnos de Odontología en el 2015, que encontró un nivel 
favorable del $100 \%$ en respuestas correctas a esta pregunta.

En cuanto al diagnóstico de paro cardiorrespiratorio, 86 alumnos $(58,9 \%)$ de quinto y sexto año respondieron correctamente; semejante al resultado de Contreras [2], en el que 42 alumnos (85,7\%) respondieron correctamente, con lo que obtuvieron un resultado más favorable.

Con respecto al diagnóstico del síncope, se obtuvo un resultado favorable, pues 101 alumnos $(69,2 \%)$ de quinto y sexto año respondieron correctamente, a diferencia del diagnóstico hallado por Contreras [2], en el que veintidós alumnos (44,9\%) respondieron correctamente, con lo que obtuvieron un resultado desfavorable.

En cuanto al diagnóstico del síndrome convulsivo, se obtuvo un resultado desfavorable, pues respondieron correctamente 61 alumnos $(41,8 \%)$ de quinto y sexto año, a diferencia del estudio de Contreras [2], en el que veintisiete alumnos $(55,1 \%)$ respondieron correctamente, con lo que obtuvieron un resultado favorable.

Con respecto al diagnóstico de reacción tóxica a anestésicos locales, se obtuvo un resultado desfavorable, pues respondieron correctamente 48 alumnos $(32,9 \%)$ de quinto y sexto año. Contreras [2] obtuvo un resultado sin diferencia significativa de trece alumnos $(26,5 \%)$ que respondieron correctamente.

En cuanto al diagnóstico de anafilaxia, se obtuvo un resultado favorable, pues respondieron correctamente ochenta alumnos $(54,8 \%)$ de quinto y sexto año. Sin embargo, Contreras [3] obtuvo un resultado más favorable con 41 alumnos (83,7\%) que respondieron correctamente. Mckenzie [20], en un estudio a odontólogos en Costa Rica en el 2005 , obtuvo un resultado desfavorable con el $40 \%$ de respuestas correctas.

Con respecto al diagnóstico de la crisis hipertensiva, se obtuvo un resultado de 85 alumnos $(58,2 \%)$ de quinto y sexto año que respondieron correctamente; a diferencia del hallado por Contreras [2], en el que obtuvo un resultado significativamente más favorable de 41 alumnos $(83,7 \%)$ que respondieron correctamente. Mckenzie [20] obtuvo un resultado favorable de $88 \%$ de respuestas correctas.

En cuanto al diagnóstico de hiperglicemia, se obtuvo un resultado favorable con 119 alumnos $(81,5 \%)$ de quinto y sexto año que respondieron correctamente. Contreras [2] obtuvo un resultado semejante en el estudio realizado en Perú, en el que 47 alumnos (95,9\%) respondieron correctamente obteniendo un resultado favorable.

Con respecto al diagnóstico de la crisis aguda del asma, se obtuvo un resultado desfavorable en el que 69 alumnos (47,3\%) de quinto y sexto año respondieron correctamente, a diferencia al hallado por Contreras [2], quien obtuvo un resultado favorable de 31 alumnos $(63,3 \%)$ que respondieron correctamente.

En cuanto al diagnóstico de la obstrucción aguda de las vías aéreas, se obtuvo un resultado favorable de 85 alumnos $(58,2 \%)$ de quinto y sexto año que respondieron correctamente; semejante al hallado por Contreras [2] en su estudio, en el que se obtuvo un resultado favorable de 33 alumnos $(67,3 \%)$ que respondieron correctamente.

Con respecto a los primeros auxilios del síncope, se obtuvo un resultado desfavorable, pues 54 alumnos $(37,0 \%)$ de quinto y sexto año respondieron correctamente; semejante al hallado por Contreras [2], quien obtuvo un resultado desfavorable de veintidós alumnos $(44,9 \%)$ que respondieron correctamente.

En cuanto al diagnóstico de los primeros auxilios de la crisis HTA, se obtuvo un resultado desfavorable, pues 56 alumnos $(38,4 \%)$ de quinto y sexto año respondieron correctamente, a diferencia del hallado por Contreras [2], que obtuvo un resultado favorable de 38 alumnos $(77,6 \%)$ que respondieron correctamente.

En cuanto a los primeros auxilios de la hiperventilación, se obtuvo un resultado desfavorable, pues 66 alumnos $(45,2 \%)$ de quinto y sexto año respondieron correctamente; semejante al hallado por Contreras [2] en su estudio, en el que se obtuvo un resultado favorable del $80 \%$ de los alumnos que respondieron correctamente.

Con respecto a los primeros auxilios de la obstrucción aguda de las vías aéreas, se obtuvo un resultado favorable, pues 76 alumnos $(52,1 \%)$, de quinto y sexto año respondieron correctamente; semejante al hallado por Contreras [2] en el estudio realizado en Perú, en el que se obtuvo un resultado favorable de cuarenta alumnos (81,6\%) que respondieron correctamente.

Con respecto a los primeros auxilios del síndrome convulsivo, se obtuvo un resultado desfavorable; solo 33 alumnos $(22,6 \%)$ de quinto y sexto año respondieron correctamente. A diferencia del hallado por Mckenzie [20], que obtuvo un resultado 
significativamente favorable con $74,29 \%$ de respuestas correctas.

En cuanto al manejo farmacológico de intoxicación por anestésico, se obtuvo un resultado desfavorable, pues 46 alumnos $(31,5 \%)$ de quinto y sexto año respondieron correctamente, a diferencia del resultado hallado por Contreras [2] en el estudio realizado en Perú, en el que se obtuvo un resultado favorable de veintisiete alumnos $(55,1 \%)$ que respondieron correctamente.

Con respecto al manejo de anafilaxia, se obtuvo un resultado desfavorable, pues 48 alumnos $(32,9 \%)$ de quinto y sexto año respondieron correctamente, a diferencia del resultado hallado por Contreras [2], en el que se obtuvo un resultado favorable de treinta alumnos $(61,2 \%)$ que respondieron correctamente.

En cuanto al manejo farmacológico de una crisis asmática, se tuvo un resultado favorable, pues 76 alumnos $(52,1 \%)$ respondieron correctamente; semejante al hallado por Contreras [2], quien obtuvo un resultado favorable de 46 alumnos (93,9\%) que respondieron correctamente. Mckenzie [20] obtuvo un resultado favorable de $77,12 \%$ de respuestas correctas.

En cuanto a los valores normales de la frecuencia respiratoria, se obtuvo un resultado desfavorable, pues 34 alumnos $(23,3 \%)$ respondieron correctamente, a diferencia de lo hallado por Mckenzie [20], quien obtuvo un resultado favorable de $71 \%$ de respuestas correctas.

En cuanto a los primeros auxilios del paciente que aspira un cuerpo extraño, se obtuvo un resultado desfavorable, pues 45 alumnos $(30,8 \%)$ respondieron correctamente, a diferencia del resultado hallado por Mckenzie [20], quien obtuvo un resultado favorable de $68,57 \%$ de respuestas correctas.

\section{Conclusiones}

De acuerdo con los resultados de este trabajo, se llegó a la conclusión de que el nivel de conocimiento de los alumnos de quinto y sexto año de la Facultad de Odontología Pierre Fauchard fue desfavorable en un 56\%, con una población mayoritariamente femenina $(87,7 \%)$ y con una franja etaria de 23 años (35,6\%). Solo el 17,8\% de alumnos participaron en algún curso de primeros auxilios, de los cuales el 6,8\% fue teórico y el 11,0\% fue teórico-práctico.

\section{Recomendaciones}

Incluir dentro de la malla curricular de la carrera de Odontología cursos teórico-prácticos sobre primeros auxilios, concienciar y sensibilizar sobre la importancia del manejo de emergencias médicas.

\section{Referencias}

[1] Mejía CR, Quezada Osoria C, Moras Ventocilla C, Quinto Porras K, Ascencios Oyarce C. Nivel de conocimientos sobre emergencias médicas en estudiantes de medicina de universidades Peruanas. Rev Peru Med Exp Salud Pública [Internet]. 2011 [citado 2016 abr 14];28(2):202-9. Disponible en: http:// www.scielo.org.pe/pdf/rins/v28n2/a06v28n2.pdf.

[2] Contreras Enríquez CM. Nivel de conocimiento sobre el manejo de situaciones de emergencia durante la consulta odontológica [tesis]. [Lima]: Universidad Nacional Mayor de San Marcos; 2015. Disponible en: http://cybertesis.unmsm.edu.pe/bitstream/ cybertesis/4596/1/Contreras_ec.pdf.

[3] Gutiérrez Lizardi P, Martínez Menchaca HR, Rivera Silva G. Importancia actual de las urgencias médicas en el consultorio dental. Revista ADM [Internet]. 2012 [citado 2016 abr 14];59(5):208-13. Disponible en: http://www.medigraphic.com/pdfs/adm/od-2012/ od125c.pdf.

[4] Stafuzza TC, Carvalho Carrar CF, Veronese Oliveira F, Ferreira Santos C, Marchini Oliveira T. Evaluation of the dentists' knowledge on medical urgency and emergency. Braz Oral Res. 2014;28(1):1-5. doi: http://dx.doi.org/10.1590/10.1590/1807-3107BOR2014.vol28.0029.

[5] Hernández Mayorga KY, Zapata Rivera FM. Correlación de la preferencia a especialidades odontológicas y nivel de conocimiento sobre el manejo de las emergencias médicas durante la consulta odontológica, en alumnos de cuarto, quinto año y personal docente de la carrera de Odontología de la UNAN-Managua en el periodo julio-octubre del año 2014 [tesis]. [Managua]: Universidad Nacional Autónoma de Nicaragua; 2015. Disponible en: http:// repositorio.unan.edu.ni/2788/1/74362.pdf. 
[6] Moreno Arroyo MP. Formación en reanimación cardiopulmonar en las facultades de Odontología públicas de España [tesis]. [Madrid]: Universidad Complutense de Madrid; 2010. Disponible en: http://eprints.ucm.es/10811/1/T31909.pdf.

[7] Sudeep CB, Sequeira PS, Jain J, Prataap N, Jain V, Maliyil M. Awareness of emergency drugs uses among students and teaching faculty in a dental college in Coorg, Karnataka. J Indian Assoc Public Health Dent.2014;12(3):185-8. doi: http://dx.doi. org/10.4103/2319-5932.144791.

[8] Gaviño Guerrero CP, RodrichTimarchi ER. Nivel de conocimiento de cirujanos dentistas sobre el manejo de emergencias médicas durante la consulta odontológica en el distrito de Chiclayo, 2015 [tesis]. [Ciclayo]: Universidad Católica Santo Toribio de Mogrovejo; 2016. Disponible en: http://dspace.uniandes.edu.ec/bitstream/123456789/162/1/ TUAODONT014-2011.pdf.

[9] Paredes Balseca JC. Protocolos para emergencias médico-odontológicas más frecuentes en la zona centro del país [tesis]. [Ambato]: Universidad Regional Autónoma de los Andes; 2011. Disponible en: http://dspace.uniandes.edu.ec/bitstream/12345678 9/162/1/TUAODONT014-2011.pdf.

[10] Torres Rivera GV. Nivel de conocimientos del manejo de urgencias médicas originadas por la administración de lidocaína con epinefrina por estudiantes de internado de Odontología de la Universidad Nacional Mayor de San Marcos en el año 2015 [tesis]. [Lima]: Universidad Nacional Mayor de San Marcos; 2015. Disponible en: http://cybertesis.unmsm. edu.pe/bitstream/cybertesis/4413/1/Torres_rg.pdf.

[11] Gonzaga HF, Buso L, Jorge MA, De Sousa Gonzaga LH, Donizetti Chaves M, Paes de Almeida O. Evaluation of knowledge and experience of dentists of São Paulo state, Brazil about cardiopulmonary resuscitation. Braz Dent J. 2013;14(3):220-2. doi: http:// dx.doi.org/10.1590/S0103-64402003000300015.

[12] Carvalho RM, Costa LR, Marcelo VC. Brazilian dental students' perceptions about medical emergencies: A qualitative exploratory study. J Dent Educ [internet]. 2008 [citado 2016 may 3];72(11):1343-9 Disponible en: http://www.jdentaled.org/content/ 72/11/1343.full.pdf+html.

[13] Mohan M, Sharma H, Parolia A, Barua A. Knowledge, attitude and perceived confidence in handling medical emergencies among dental practitioners in Dakshina Kannada, India. oHDM [internet]. 2015 [citado 2016 sep 7];14(1):27-31. Disponible en: http://www.omicsonline.com/open-access/know ledge-attitude-and-perceived-confidence-in-handl ing-medical-emergencies-among-dental-practitioners-2247-2452-14-760.pdf.
[14] Benavides M. Emergencias médicas que se han presentado en los consultorios odontológicos de la Región Huetar Norte y competencias requeridas para su manejo [tesis]. [San José]: Universidad Latinoamericana de Ciencia y Tecnología; 2010. Disponible en: http://bb9.ulacit.ac.cr/tesinas/publicaciones/040453.pdf.

[15] Ehigiator O, Ehizele AO, Ugbodaga PI. Assessment of a group of Nigerian dental students' education on medical emergencies. Ann Med Health Sci Res. 2014;4(2):248-52. doi: http://dx.doi.org/10. 4103/2141-9248.129052.

[16] Tanupriya G, Aradhya S. Preparedness for Management of Medical Emergencies Among Dentists in Udupi and Mangalore, India. J Contemp Dent Pract [Internet]. 2008 [citado 2016 ago 2]; 9(5):929. Disponible en: http://www.jaypeejournals.com/ eJournals/ShowText.aspx?ID=1903\&Type=FREE $\& \mathrm{TYP}=\mathrm{TOP} \& \mathrm{IN}=\_$eJournals/images/JPLOGO. gif\&IID=161\&isPDF=YES.

[17] Newby JP, Keast J, Adam WR. Simulation of medical emergencies in dental practice: Development and evaluation of an undergraduate training programme. Australian Dental. 2010;55(4):399-404. doi: https:// doi.org/10.1111/j.1834-7819.2010.01260.x.

[18] Villena Villa KM. Nivel de conocimiento sobre emergencias médicas durante la consulta odontológica de los estudiantes de quinto y sexto año de Estomatología de la Universidad Nacional de Trujillo, 2013 [tesis]. [Trujillo]: Universidad Nacional de Trujillo; 2013. Disponible en: http://dspace.unitru. edu.pe/xmlui/bitstream/handle/123456789/613/ VillenaVilla_K.pdf? sequence $=1$ \&isAllowed $=\mathrm{y}$.

[19] Elanchezhiyan S, Elavarasu S, Vennila K, Renukadevi R, Nazargi Mahabob M, Sentilkumar B, et al. Awareness of dental office medical emergencies among dental interns in Southern India: An analytical study. J Dent Educ [Internet]. 2012 [citado 2016 ago 22]; 77(3):364-9. Disponible en: http://www. jdentaled.org/content/77/3/364.full.pdf+html.

[20] Mckenzie SE. Nivel de conocimiento en el manejo de emergencias médicas durante la consulta dental en la provincia de Limón [tesis]. [San José]: Universidad Latinoamericana de Ciencia y Tecnología; 2005. Disponible en: http://bb9.ulacit.ac.cr/tesinas/ publicaciones/031094.pdf.

[21] Jodalli PS, Ankola AV. Evaluation of knowledge, experience and perceptions about medical emergencies amongst dental graduates (interns) of Belgaum City, India. J Clin Exp Dent. 2012;4(1):e14-8. doi: http://dx.doi.org/10.4317/jced.50627. 
[22] Arsati F, Montalli VA, Martão Flório F, Cama Ramacciato J, Lopes da Cunha F, Cecanho R, et al. Brazilian dentists' attitudes about medical emergencies during dental treatment. J Dent Educ [Internet]. 2010 [citado 2016 sep 7];74(6):661-6. Disponible en: http://www.jdentaled.org/content/74/6/661.full. pdf + html.

[23] Shenoy N, Ahmed J, Ongole R, Boaz K, Srikant N. Are dental surgeons prepared for medical emergencies. IJBR. 2013;4(9):461-4. doi: http://dx.doi.org/10. 7439/ij.

[24] Muller MP, Stehr SN. A state-wide survey of medical emergency management in dental practices: Incidence of emergencies and training experience. Emerg Med J. 2008;25(2):296-300. doi: http://dx. doi.org/ 10.1136/emj.2007.052936.

[25] Uribe J, Viniegra L. Evaluación de aptitudes clínicas ante complicaciones médico-dentales. Rev Med IMSs [Internet]. 2004 [citado 2016 may 17];42(1):1120. Disponible en: http://www.medigraphic.com/ pdfs/imss/im-2004/im041c.pdf.

[26] Stoeva I. The asses of dental students' knowledge and skills in management of medical emergencies in dental office. Journal of Iмав [Internet]. 2011 [citado 2016 jun 23];17(2):132-3. Disponible en: http://citeseerx.ist.psu.edu/viewdoc/summary?doi $=10.1 .1 .390 .6157$.
[27] Tupiza Guerrero EM. Conocimiento sobre shock anafiláctico y su manejo odontológico de los alumnos de séptimo, octavo y noveno semestre de la Facultad de Odontología de la Universidad Central del Ecuador. Período 2015-2016 [tesis]. [Quito]: Universidad Central del Ecuador; 2016. Disponible en: http://www.dspace.uce.edu.ec/bitstream/25000/6803/1/T-UCE-0015-358.pdf.

[28] Broadbent JM, Murray Thomson W. The readiness of New Zealand general dental practitioners for medical emergencies. N Z Dent J [Internet]. 2001 [citado 2016 ago 22]; 97:81-6. Disponible en: https:// www.researchgate.net/profile/William_Thomson2/ publication/11659475_The_readiness_of_New_Zealand_general_dental_practitioners_for_medical_ emergencies/links/02e7e51885521550be000000.pdf.

[29] Bilich LA, Jackson SC, Bray BS, Willson MN. High-fidelity simulation: Preparing dental hygiene students for managing medical emergencies. J Dent Educ [Internet]. 2015 [citado 2016 ago 22]; 79(9):1074-81. Disponible en: http://www.jdentaled.org/content/79/9/1074.full.pdf+html.

[30] Mutzbauer TS, Rossi R, Ahnefeld FW, Sitzmann F. Emergency medical training for dental students. Anesth Prog [Internet]. 1996 [citado 2016 sep 7]; 43(2):37-40. Disponible en: https://www.ncbi.nlm. nih.gov/pmc/articles/PMC2148788/. 